\title{
Corrección de eventos dispersos en algoritmos de reconstrucción tomográfica para PET
}

\section{Scatter correction in PET tomographic reconstruction algorithms}

Presentación: 6 y 7 de Octubre de 2020

\section{Doctorando:}

\section{Mariana Prieto Canalejo}

Grupo de Inteligencia Artificial y Robótica (GIAR), Facultad Regional Buenos Aires, Universidad Tecnológica Nacional - Argentina mprietocanalejo@frba.utn.edu.ar

\section{Director/es:}

\section{Daniel Minsky}

\section{Resumen}

La Tomografía por Emisión de Positrones (PET) permite cuantificar el metabolismo celular, siendo una de las técnicas más poderosas en el diagnóstico precoz de enfermedades cardíacas, oncológicas y neurológicas. Las imágenes se obtienen mediante la administración de un radiofármaco que marca el proceso metabólico a estudiar. Los rayos gamma que emite el radiotrazador, inciden en detectores ubicados alrededor del paciente generando eventos que se almacenan para la reconstrucción de la imagen. Para obtener imágenes cuantificables, se realizan diversas correcciones a los eventos, entre ellas por eventos dispersos. Con el objetivo de implementar la corrección por eventos dispersos, se realiza, en primera instancia, el modelado de los mismos por medio del algoritmo Single Scatter Simulation (SSS) obteniendo una estimación de la dispersión. Posteriormente se realiza la corrección en la imagen de emisión. Para la corrección se incorpora al algoritmo de reconstrucción MLEM (maximum-likelihood expectation-maximization) la estimación obtenida con el modelado. Los resultados preliminares, obtenidos en simulaciones de volúmenes de actividad constante, han demostrado que la implementación realizada mejora la homogeneidad de la imagen de emisión.

Palabras clave: Tomografía por Emisión de Positrones, Single Scatter Simulation, corrección de eventos dispersos.

\begin{abstract}
Positron Emission Tomography (PET) allows quantification of cellular metabolism, and it is one of the most powerful techniques in early diagnosis of heart, oncological and neurological diseases. Images are obtained through the administration of a radiopharmaceutical that marks the metabolic process that will be studied. Gamma rays emitted by radiotracer fall upon detectors set around the patient generating events that are stored for image reconstruction. In order to get quantifiable images, different
\end{abstract}


corrections are made to events, including scatter. With the aim of implement correction by scatter, modeling through Single Scatter Simulation (SSS) algorithm is made at first, getting estimated scatter. Afterwards, correction in emission image is made. For correction, estimation made through modeling is incorporated to reconstruction algorithm MLEM (maximum-likelihood expectation-maximization). Preliminary results, obtained in constant activity volumes simulations, have shown that implementation made improves emission image homogeneity.

Keywords: Positron Emission Tomography, Single Scatter Simulation, scatter correction.

\section{Introducción}

En la técnica de Tomografía por Emisión de Positrones, el radiofármaco administrado al paciente genera un fenómeno de aniquilación que produce dos rayos gamma colineales de $511 \mathrm{keV}$. Estos rayos se originan en un mismo punto pero en sentidos opuestos permitiendo dibujar una línea en el espacio llamada Línea de Respuesta (LOR por sus siglas en inglés). Los algoritmos de reconstrucción permiten a partir de dichas LORs generar imágenes en las que los puntos brillantes se corresponden con zonas de mayor acumulación del radiofármaco, debido a una actividad celular mayor como por ejemplo un tumor. Los puntos más oscuros, por otro lado, corresponden a una actividad celular menor a la normal.

Los rayos gamma que atraviesan el cuerpo del paciente sufren dispersión Compton después de la interacción con los tejidos que atraviesan. Esto resulta en un cambio de dirección de los rayos y una pérdida parcial de energía, generando LORs erróneas. La presencia de la componente de dispersión se manifiesta como una pérdida de resolución espacial y como una aparente migración de actividad de las regiones oscuras a las brillantes (Zaidi \& Montandon, 2007). Para mejorar la calidad de la imagen de emisión es necesario aplicar dicha corrección.

En este trabajo se aborda el análisis e implementación de algoritmos de modelado de eventos dispersos. Se desarrolla el algoritmo de modelado de eventos dispersos basado en Single Scatter Simulation (SSS) diseñado por Watson et al (1996), incorporando la segmentación por energía (Harrison et al, 1991)(Brusaferri at al, 2018). Este algoritmo es rápido, altamente paralelizable y es simple su incorporación a los algoritmos de reconstrucción (Bentourkia \& Sarrhini, 2004)(Werling at al, 2000)

Se añade el modelado de eventos dispersos a la reconstrucción de la imagen de emisión para realizar la corrección de los mismos (Cheng et al, 2007). El algoritmo de reconstrucción utilizado es el MLEM, donde el estimador ML permite encontrar el conjunto de parámetros de una población que se corresponden con una muestra dada.

Estos algoritmos serán incorporados al algoritmo de reconstrucción de AR-PET (AR-PET, 2018) potenciando el desarrollo de dicho proyecto.

\section{Desarrollo}

\subsection{Segmentación de eventos dispersos}

Al sufrir efecto Compton los eventos reducen su energía dada por la ecuación (1). Por ello segmentar las LORs que poseen menor energía permite reducir la cantidad de datos a 
procesar así como también seleccionar los eventos que tienen mayor probabilidad de haber sufrido dispersión.

$$
E^{\prime}=\frac{E_{0}}{1+\frac{E_{0}}{m_{e} c}(1-\cos (\theta))}
$$

Se realiza el análisis de energías de eventos dispersos comparados con los que no sufrieron dispersión. Para ello se utilizan en simulaciones realizadas en GATE (Strulab at el, 2003), con la definición de la geometría de AR-PET, las cuales realizan un seguimiento del rayo e indican cuales sufrieron dispersión.

Como se observa en la figura 1 los eventos que sufren dispersión poseen una distribución de energías por debajo de los que no la sufren. Dado que las LORs seleccionadas para realizar la reconstrucción son las que se encuentran en la banda de energía 470-540 Kev se tomará para la estimación de scatter las LORs que se encuentren en el rango 440-500 Kev.

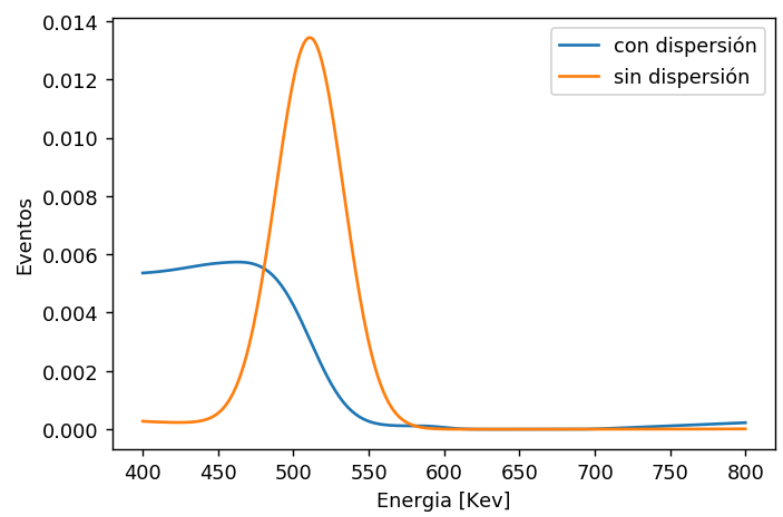

Figura 1:Distribución de eventos en energías

\subsection{Modelización de eventos dispersos}

A partir del modelo que se observa en la figura 2, el algoritmo integra la contribución de la dispersión de todos los puntos de dispersión Ps cuando la emisión proviene de una LOR determinada por los puntos $\mathrm{P}_{\mathrm{A}}$ y $\mathrm{P}_{\mathrm{B}}$. Dichos puntos Ps son distribuidos aleatoriamente dentro del campo de visión.

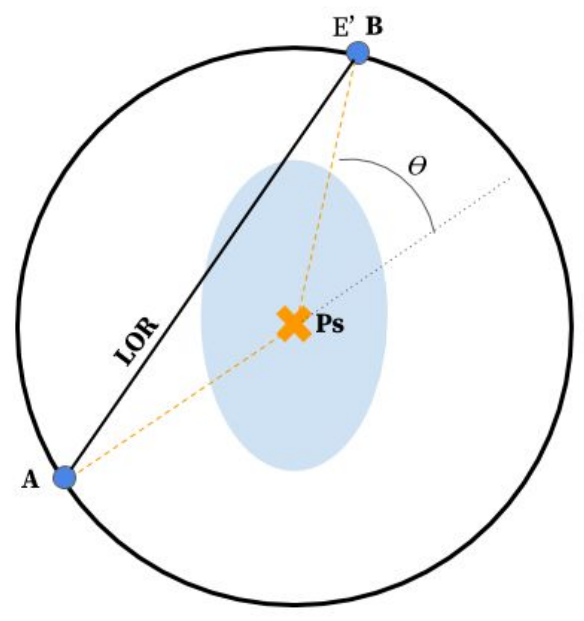

Figura 2: Modelo de dispersión 
La contribución de la dispersión se basa en la probabilidad que se haya dado dicha dispersión, esta probabilidad está dada por la sección eficaz de dispersión $(\sigma c)$, dada por la ecuación de Klein y Nishina en conjunto con el mapa de actividad y de atenuación.

La ecuación de SSS está dada por:

$$
S_{A B}=\int_{V S} d V S \frac{\sigma_{A S} \sigma_{B S}}{4 \pi R_{A S}^{2} R_{B S}^{2}} \frac{\mu(E)}{\sigma_{0}} \frac{d \sigma_{0}(E, \theta)}{d \Omega}\left[I_{A}+I_{B}\right]
$$

Dónde:

$$
\begin{gathered}
I_{A}=\varepsilon_{A S}(E) \epsilon_{B S}\left(E^{\prime}\right) e^{-\left(\int_{S}^{A} \mu(E) d s+\int_{S}^{B} \mu\left(E^{\prime}\right) d s\right)} \int_{S}^{A} \lambda d s \\
I_{B}=\varepsilon_{B S}(E) \epsilon_{A S}\left(E^{\prime}\right) e^{-\left(\int_{S}^{B} \mu d s^{\prime}+\int_{S}^{A} \mu\left(E^{\prime}\right) d s\right)} \int_{S}^{B} \lambda d s
\end{gathered}
$$

Donde, E y E denotan las energías de los fotones antes y después de la interacción Compton en función del ángulo de dispersión $\theta \cdot \frac{d \sigma_{0}}{d \Omega}$ es la sección eficaz diferencial para la dispersión. $\lambda$ la distribución de emisión en el objeto. $\mu$ el coeficiente de atenuación del objeto a la energía del fotón. $\sigma \mathrm{A}$ y $\sigma \mathrm{B}$ son las secciones eficaces geométricas del detectores A y $\mathrm{B}$ respectivamente. $\varepsilon \mathrm{A}$ y $\varepsilon \mathrm{B}$ son las eficiencias del detector, dependiendo de la energía del fotón, la resolución de la energía y la dirección $\overline{A S}$ y $\overline{B S}$ respectivamente.

Segmentando las LORs con las energías mencionadas, se implementa el algoritmo de modelado con la estimación de eventos dispersos que se propone en la ecuación (2).

\subsection{Corrección de eventos dispersos}

Para la corrección de eventos dispersos se incorpora la estimación obtenida con el algoritmo SSS al algoritmo de reconstrucción iterativa MLEM en modo lista:

$$
x_{j}^{(n+1)}=\frac{x_{j}^{(n)}}{\sum_{i=1}^{N} C i j} \sum_{i=1}^{M} \frac{C i j}{\sum_{j^{\prime}=1}^{M} C i j x_{j^{\prime}}^{(n)}}
$$

Donde $X_{j}$ es el valor reconstruido del j-ésimo vóxel de la imagen en la que con $\mathrm{K}$ vóxeles a reconstruir, $\mathrm{M}$ es el número de LORs, $\mathrm{Cij}$ denota la probabilidad de que un evento del j-ésimo vóxel se detecte en la i-ésima LOR, $\mathrm{N}$ es el número total de eventos detectados. $\mathrm{El}$ factor de probabilidad se calcula como la longitud del rayo desde la LOR que se cruza con el vóxel con el algoritmo Siddon (1985) en conjunto con la atenuación que sufre dicha LOR y la estimación de eventos dispersos calculada. 


\subsection{Resultados}

Para la evaluación del desarrollo implementado se realizan simulaciones de volúmenes de actividad constante en GATE con la geometría de AR-PET. Dicho volumen es definido como un cilindro de $30 \mathrm{~cm}$ de diámetro y $8 \mathrm{~cm}$ de alto.

En la figura 3 se puede observar la imagen reconstruida con y sin corrección, en esta se observa que la homogeneidad de la imagen corregida es $60 \%$ mayor.

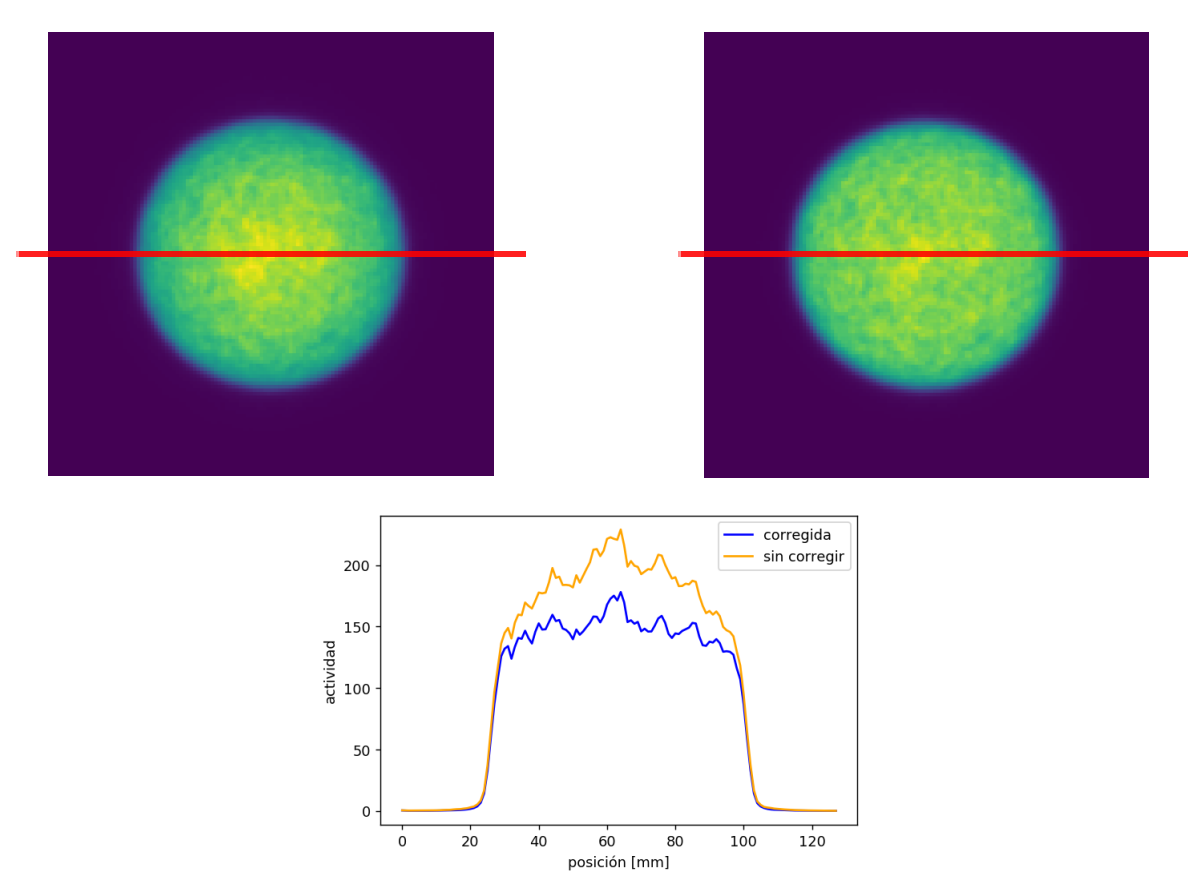

Figura 3: (a) Imagen sin corregir. (b) imagen corregida. (c) corte transversal de ambas imágenes.

\section{Conclusiones}

En este trabajo se ha presentado la implementación de algoritmos para corrección de eventos dispersos, obteniendo una mejora en la homogeneización de la imágen del $60 \%$ en una imagen de actividad constante. Se continuará con el testeo de los algoritmos con volúmenes que posean variación de actividad, dando lugar a la evaluación de los algoritmos con imágenes más complejas. A su vez se realizará el análisis de tiempo de procesamiento.

Una vez obtenida una correcta modelización de los eventos dispersos se comenzará de cálculo del mapa de atenuación con dicha modelización (Berker \& Li, 2016)

\section{Referencias}

H. Zaidi and M. Montandon. ( 2007) Scatter compensation techniques in PET. PETClin. 2, 219-234. 
C. Watson, D. Newport, and M. Casey. (1996). A single scatter simulation technique for scatter correction in 3D PET. Three-dimensional image reconstruction in radiology and nuclear medicine. Springer, S. 255-268.

C. C. Watson,New, faster, image-based scatter correction for 3D PET.IEEE Transactions on Nuclear Science 47, Nr. 4, S. 1587-1594, 2000

C. C. Watson, M. E. Casey, C. Michel, and B. Bendriem, Advances in scatter correction for 3D PET/CT. IEEE NSS / MIC, vol. 5, pp. 3008-3012,2004

R. Harrison, D. Haynor, and T. Lewellen. (1991) Dual energy window scatter corrections for positron emission tomography. Conf. Rec. of IEEE Nuc. SciSymp. Santa Fe N.M. pp. 1700-1704.

L. Brusaferri, A. Bousse, N. Efthimiou, E. Emond, D. Atkinson, S. Ourselin, B. Hutton, S. Arridge and K. Thielemans (2018) Maximum-likelihood estimation of emission and attenuation images in 3D PET from multiple energy window measurements. IEEE NSS/MIC Conference Proceedings.

M. Bentourkia and O. Sarrhini. (2004). Simultaneous Attenuation and Scatter Correction by Restoration in PET imaging. IEEE Symposium ConferenceRecord Nuclear Science.

Werling, A., Bublitz, O., Doll, J., Adam, L.-E., and Brix (1999). Fast implementation of the single scatter simulation algorithm and its use in iterative image reconstruction of PET data. 1999 IEEE Nuclear Science Symposium. Nuclear Science Symposium and Medical Imaging Conference.

Cheng, J.-C., Rahmim, A., Blinder, S., Camborde, M.-L., Raywood, K., \& Sossi, V. (2007). A scatter-corrected list-mode reconstruction and a practical scatter/random approximation technique for dynamic PET imaging. Physics in Medicine and Biology, 52(8), 2089-2106.

AR-PET. (2018). Ministerio de Desarrollo Productivo. Consultada el 11 de agosto de 2020 https://www.argentina.gob.ar/noticias/el-tomografo-ar-pet-hecho-por-cnea-ya-es ta-en-el-hospital-de-clinicas.

D. Strulab, G. Santin, D. Lazaro, D. Breton, y C. Morel, "GATE (geant4 application for tomographic emission): a PET/SPECT general-purpose simulation platform," Nuclear Physics B - Proceedings Supplements, vol. 125, pp. 75-79, Septiembre 2003.

R. L. Siddon (1985) "Fast Calculation of the Exact Radiological Path for a Three-dimensional CT," J. Med. Phys., vol. 12, no. 2, pp. 252-255.

Y. Berker and Y. Li. (2016). Attenuation correction in emission tomography usingthe emission data - A review. Medical Physics Bd. 43 PMC, S. 807-832.

Berker, Y., Maier, J., \& Kachelries, M. (2018). Deep Scatter Estimation in PET: Fast Scatter Correction Using a Convolutional Neural Network. 2018 IEEE Nuclear Science Symposium and Medical Imaging Conference Proceedings (NSS/MIC). 